\title{
Should you count your mice before they're weaned?
}

In common with the practice at many other institutions, the animal facility at Great Eastern University did not include neonatal mice in the census count until they were weaned. It was also common for investigators to euthanize transgenic mice that did not have the needed genotype for a particular research project. These two practices in combination provided an opportunity for some researchers to avoid paying per diem charges on their superfluous transgenic mice by euthanizing them before they were weaned. Of course, because the animals were never counted on the census, they also were never subtracted from the number of animals approved by the IACUC for that study. This chain of events never raised any eyebrows at Great Eastern until the Committee's unaffiliated member nonchalantly asked about the need to count the tadpoles that were part of another study.

"I was wondering," said Pat Waite, "if we're supposed to count tadpoles as 'animals' or if we're supposed to wait until they pretty much change into frogs?"

"That's a good question" replied Larry Cristofini, the IACUC Chairman. "Since tadpoles are, in the IACUC's opinion, self-sustaining animals, we have always counted them as soon as they are capable of feeding themselves. In other words, similar to what we do for zebrafish fry."

"Okay," said Waite, "but that leads me to another question. If we count tadpoles as soon as they are capable of feeding themselves, how come we don't count mouse pups, including transgenic pups, as soon as they are born? They're perfectly capable of nursing their dams at that time. Not only that, but I've been told many times that pups start eating solid food by themselves before they are weaned. Don't we have two standards? Shouldn't we really put mouse pups on the census as soon as they're born?"

"Don't worry about it, Pat" said Cristofini. "We've always done it this way and we've never had any complaints from OLAW [Office of Laboratory Animal Welfare], nor have I ever seen anything that OLAW has written to contradict what we do. Not only that, but when we have our AAALAC [Association for Assessment and Accreditation of Laboratory Animal Care International] site visits, nobody has ever questioned us. I think we can take that to mean that we're in compliance with federal regulations."

Is Great Eastern in compliance with their method of accounting for neonatal mice, or are OLAW and AAALAC just not making an issue of a common practice?

\section{RESPONSE}

\section{Pool the pups}

\section{H. Hugh Harroff, Jr., DVM, DACLAM \& David G. Watson, PhD}

The unaffiliated member of the Great Eastern IACUC has a valid point in thinking that the mouse pups in this situation should be counted in the animal census from the time of birth. We believe that the IACUC has three issues to address in this situation.

First, should the investigator pay per diem charges for all of the mouse pups born to the dams on the study, even though they may not be genetically suitable for inclusion in the study? This is a minor issue for which there can be local answers without regard to regulatory considerations. We believe the cost of housing the extra pups is negligible and should be covered by the 'overhead' or operating funds of the institution.

Second, should the census include all of the mouse pups born to the dams on the study? As stated earlier, we concur with Waite (the unaffiliated member) that there is an inconsistency in the institution's counting of animals (tadpoles versus mouse pups) and adding them to the census. We suggest that a satisfactory solution would be for the Great Eastern IACUC to generate and approve a generic protocol for animals being held in a 'pool' pending assignment to a research study. The investigator on this protocol would be a member of the University's Laboratory Animal Resources. The protocol would cover all species that might be held pending future assignment to a study, including the mouse pups; it would also specify procedures for animal care and treatment and would describe in detail the planned tests (e.g., genetic monitoring, complete blood count, serum chemistry) in determining suitability of the animals for assignment to research studies. Finally, the protocol would specify the disposition of unsuitable animals such as euthanasia and the method of euthanasia. At the time of birth all of the mouse pups would enter this 'pool' protocol, which would automatically include them in the animal census. Because genetic monitoring would be a decisive issue in determining the suitability of the mouse pups, the protocol would probably fall into USDA pain category $\mathrm{C}$ or $\mathrm{D}$.

Third, should all of the mouse pups born to the dams assigned to the study be counted against the total allotted by the IACUC for the protocol, even though genetic monitoring may eventually determine that many are unsuitable for the study? It is absolutely not permissible to exceed the number of animals allotted to a protocol by the IACUC. We believe that the 'pool' protocol described earlier would also solve this problem. With the 'pool' protocol, there would be a description of the criteria used to determine a 